\title{
FISCHER DECOMPOSITION FOR MASSLESS FIELDS OF SPIN 1 IN DIMENSION 4
}

\author{
F. BRACKX* ${ }^{*}$ H. DE SCHEPPER* ${ }^{*}$, L. KRUMP $\ddagger$ \& V. SOUČEK ${ }^{\ddagger}$
} ${ }^{*}$ Clifford Research Group, Department of Mathematical Analysis, Faculty of Engineering
and Architecture, Ghent University, Belgium
$\ddagger$ Charles University in Prague, Faculty of Mathematics and Physics, Mathematical Insti-
tute, Sokolovská 83, 18675 Praha, Czech Republic

\begin{abstract}
The massless field equations for lower integer and half-integer values of spin in Minkowski space are fundamental equations in mathematical physics. Their counterpart in Euclidean spacetime is a system of elliptic equations, which was already studied from the viewpoint of function theory in the framework of so-called Hodge systems for differential forms of various degrees. In dimension 4 it is possible to substitute spinor calculus for the usual tensor notation. In the present paper we concentrate on the case of the massless field equation for spin 1 in dimension 4 , and we treat, in a spinor formalism, a fundamental concept of its function theory: the Fischer decomposition of polynomial spinor fields, for which we give simple and independent proofs.
\end{abstract}

\section{INTRODUCTION}

The field equations for massless particles of integer and half-integer spin in Minkowski space are fundamental equations in theoretical particle physics. Solutions of these equations in dimension 4 were carefully and systematically studied as part of Penrose's twistor programme (see $[9,10,14]$ ). In dimension 4 for each value of spin there is one equation which comes in two possible versions: for primed and for unprimed spinor fields.

The masses field equations were generalised to higher dimension, in the framework of Clifford analysis, in $[12,13]$. Classical Clifford analysis deals with the so-called monogenic function theory of solutions of the Dirac equation in the $m$-dimensional Euclidean space $\mathbb{R}^{m}$ for fields taking their values in a Clifford algebra, say $\mathbb{C}_{m}$, or in the irreducible $\mathbb{C}_{m}-$ module $S$. Recently a lot of attention has been paid to the properties of special classes of those monogenic solutions having their values restricted to the space of $k$-vectors, see, e.g., $[4,1,8,2,6,7]$. In this case the Dirac equation reduces to the so-called Hodge-de Rham system, which becomes the Riesz system when $k=1$.

For general dimension and general spin, the massless field equations on the one side and the Hodge-de Rham systems on the other are clearly different systems, because their solutions have values in different irreducible $\operatorname{Spin}(m)$-modules. However there is a particular case where relations between both systems are possible: the dimension should be even, say $m=2 n$, spin should be 1 and the Hodge-de Rham systems should be considered for forms of degree equal to the half dimension $n$. More precisely (see $[12,6]$ ), we shall use the classification of irreducible representation of the Lie group $\operatorname{Spin}(n), n=2 m$ by highest weights $\lambda=\left(\lambda_{1}, \ldots \lambda_{m}\right)$. The Hodge-de Rham systems are systems of first order PDE's for fields with values in irreducible representation $V_{\lambda_{k}}, k=1, \ldots, m-1$, where $\lambda_{k}=(1, \ldots, 1,0 \ldots, 0)$ with $k$ nontrivial entries, resp. $\lambda_{m, \pm}, \lambda_{m, \pm}=(1, \ldots, 1, \pm 1)$. 
Massless fields equations are defined for fields with values in irreducible representations $V_{\lambda_{s}}, s=1,2,3, \ldots$, where $\lambda_{s, \pm}=(s / 2, \ldots, s / 2, \pm s / 2)$. Hence both types of fields can be compared only for the weights $\lambda=(1, \ldots, 1, \pm 1)$, i.e., for $k=m$ (the Hodge-de Rham case) and $s=2$ (massless field case).

The simplest and at the same time the most important case for theoretical physics is the case of spin 1 in dimension 4, where the space of 2-vectors (or 2-forms) splits into a selfdual and an anti-selfdual part having the highest weights $\lambda_{ \pm}=(1, \pm 1)$. The corresponding systems of equations then are equivalent to the Euclidean version of the Maxwell equations. This is the case treated in the paper.

The Fischer decomposition for general Hodge-de Rham (and Moisil-Teodorescu) systems was studied in [7]. A Fisher decomposition is a direct sum decomposition of a space of polynomial null solutions to a differential operator in terms of irreducible representations for the invariance group of the operator considered. The purpose of the present paper is to describe in detail the Fischer decomposition of the space of polynomial solutions to the spin 1 massless field equation in the 4-dimensional Euclidean space, using the language of spinor fields. Spinor language indeed is a very suitable tool for the treatment of selfdual and anti-selfdual forms in dimension 4 and it makes the presentation of the results much more transparent. Throughout the text we will freely use standard notations from spinor calculus in dimension 4 (see [10]). Also the identification of spin 1 massless fields with 1- and 2-forms and the relations between the massless field equations and the Hodge-de Rham systems will be reviewed, and simple and independent proofs of the Fischer decomposition in this particular setting will be given. Note that the Fischer decomposition for the selfdual case of spin 1 massless fields was already stated, not proven, in [3].

In order to make the paper self-contained a preliminary section containing the necessary definitions and notations is included.

\section{Preliminaries}

2.1. Spin(4)-modules. In this section we recall some basic notations and definitions from spinor analysis in dimension 4, for the case of a Euclidean real form and its representations. For a detailed treatment we refer to the monograph [10], which however focusses on the Lorentz group as a real form of $\mathrm{SO}(4, \mathbb{C})$, an important case for theoretical physics.

Let $E=\mathbb{R}^{4}$ be the Euclidean vector space of dimension 4 and let $E_{c}=\mathbb{C}^{4}$ be its complexification. The corresponding complex Clifford algebra $\mathbb{C}_{4}$ is isomorphic to the matrix algebra $\operatorname{Mat}_{4}(\mathbb{C})$ of $4 \times 4$ complex matrices. Up to an isomorphism, there is a unique irreducible representation $S \simeq \mathbb{C}^{4}$ of $\mathbb{C}_{4}$, which can be realized as the space of column vectors subject to the left action of $\operatorname{Mat}_{4}(\mathbb{C})$. The even part $\mathbb{C}_{4}^{+}$of the Clifford algebra $\mathbb{C}_{4}$ is realized by the set of all diagonal two by two block matrices, hence the representation $S$ decomposes under the action of $\mathbb{C}_{4}^{+}$as the direct sum $S=S_{A} \oplus S^{A^{\prime}}$ of two irreducible $\mathbb{C}_{4}^{+}$-modules. By convention, the space $S_{A}$ is a module for the action of the upper left block while $S^{A^{\prime}}$ is a module for the action of the bottom right block. As vector spaces, $S_{A}$ and $S^{A^{\prime}}$ are isomorphic to $\mathbb{C}^{2}$.

In dimension 4 the group $\operatorname{Spin}(4, \mathbb{C})$ is isomorphic to $\operatorname{SL}(2, \mathbb{C}) \times \operatorname{SL}(2, \mathbb{C})$ and it can be realized as a subgroup of $\mathbb{C}_{4}^{+}$, where the first (respectively second) factor is supposed to be a subgroup of the upper left (respectively bottom right) block and both $S_{A}$ and $S^{A^{\prime}}$ are realized as the space of column vectors. The action of an element $g=\left(g_{1}, g_{2}\right) \in$ $\mathrm{SL}(2, \mathbb{C}) \times \mathrm{SL}(2, \mathbb{C})$ is given by matrix multiplication by $g_{1}$ on $S_{A}$, resp. by $g_{2}$ on $S^{A^{\prime}}$. By tradition, $A \in\{0,1\}$ and $A^{\prime} \in\left\{0^{\prime}, 1^{\prime}\right\}$. We also have to consider the dual (contragradient) modules $S^{A}:=\left(S_{A}\right)^{*}$ and $S_{A^{\prime}}:=\left(S^{A^{\prime}}\right)^{*}$, which, by convention, are realized as row vectors with the action given by right multiplication by the inverse elements $g_{1}^{-1}$, resp. $g_{2}^{-1}$. 
In what follows, we will use the Einstein convention for sums over upper and lower indices.

The modules $S_{A}$ and $S^{A}$ are selfdual, i.e. they are isomorphic as $\operatorname{Spin}(4, \mathbb{C})$-modules, and so are $S_{A^{\prime}}$ and $S^{A^{\prime}}$. We define these isomorphisms by

$$
s^{A}=\varepsilon^{A B} s_{B}, \quad s_{B}=-s^{A} \varepsilon_{A B}, \quad \text { with } \quad s^{A} \in S^{A} \quad \text { and } \quad s_{B} \in S_{B}
$$

and similarly for the primed indices, where

$$
\varepsilon_{A B}=\varepsilon^{A B}=\varepsilon_{A^{\prime} B^{\prime}}=\varepsilon^{A^{\prime} B^{\prime}}=\left(\begin{array}{cc}
0 & 1 \\
-1 & 0
\end{array}\right)
$$

It also holds that

$$
\varepsilon_{A B} \varepsilon^{B C}=\varepsilon_{A}^{C}=-\varepsilon_{A}^{C}=\delta_{A}^{C}
$$

and similar relations with primed indices. So, for example, if

$$
s_{A}=\left(\begin{array}{l}
s_{0} \\
s_{1}
\end{array}\right)
$$

then

$$
s^{A}=\left(\begin{array}{ll}
s^{0} & s^{1}
\end{array}\right)=\left(\begin{array}{ll}
s_{1} & -s_{0}
\end{array}\right)
$$

It is also useful to notice some consequences of the relations (1)(2)(3). First, for any antisymmetric element $\varphi_{A B}=\varphi_{[A B]}$ one has

$$
\varphi_{A B}=\frac{1}{2} \varphi_{C}^{C} \varepsilon_{A B}
$$

and a similar relation holds whenever two antisymmetric indices $A, B$ appear in a longer array of indices. Secondly it also holds that

$$
\varphi_{A} \psi^{A}=-\varphi^{A} \psi_{A}
$$

Note that the element $\varepsilon_{A B}$ is invariant in $\Lambda^{2}\left(S_{A}\right) \simeq \mathbb{C}$, which is the trivial representation of $S L(2, \mathbb{C})$. Hence the contraction with $\varepsilon_{A B}$ leads to an isomorphism of $\operatorname{Spin}(4, \mathbb{C})$-modules.

Two half-spinor representations $S_{A}, S^{A^{\prime}}$ and their duals generate, by tensor product, the whole ring of tensor-spinors. The key role is played by symmetric powers, due to the fact that already the second outer power is the trivial representation. Irreducible $\operatorname{Spin}(4, \mathbb{C})$-modules are classified by a couple $(a, b)$ of nonnegative integers and realized as $\odot^{a}\left(S_{A}\right) \otimes \odot^{b}\left(S_{A^{\prime}}\right)$. The standard notation for $\odot^{a}\left(S_{A}\right)$ is $S_{(A \ldots E)}$, the number of indices being equal to $a$, and similarly for the primed version. The module $\odot^{a}\left(S_{A}\right) \otimes \odot^{b}\left(S_{A^{\prime}}\right)$ is denoted by $S_{(A \ldots E)\left(A^{\prime} \ldots F^{\prime}\right)}$. Similarly, antisymmetric powers are denoted by square brackets, e.g., $\varepsilon_{A B}$ belongs to the one-dimensional module $S_{[A B]}$.

So, for example,

$$
S_{A} \otimes S_{B} \simeq S_{(A B)} \oplus S_{[A B]} \simeq S_{(A B)} \oplus \mathbb{C}
$$

and

(6) $S \otimes S=S_{A B} \oplus S_{A}^{B^{\prime}} \oplus S_{B}^{A^{\prime}} \oplus S^{A^{\prime} B^{\prime}}=S_{(A B)} \oplus S_{[A B]} \oplus S_{A}^{B^{\prime}} \oplus S_{B}^{A^{\prime}} \oplus S^{\left(A^{\prime} B^{\prime}\right)} \oplus S^{\left[A^{\prime} B^{\prime}\right]}$

2.2. Relations between vectors and spinors in dimension 4. The space of (complexified) 1 -forms $V_{a}^{\mathbb{C}} \simeq \mathbb{C}^{4}, a=0,1,2,3$, can be identified, as a $\operatorname{Spin}(4, \mathbb{C})$-module, with the tensor product $S_{A A^{\prime}}=S_{A} \otimes S_{A^{\prime}}$ by

$$
x_{A A^{\prime}}=\left(\begin{array}{ll}
x_{00^{\prime}} & x_{01^{\prime}} \\
x_{10^{\prime}} & x_{11^{\prime}}
\end{array}\right) \simeq \frac{1}{\sqrt{2}}\left(\begin{array}{rr}
v_{0}+i v_{3} & v_{1}+i v_{2} \\
-v_{1}+i v_{2} & v_{0}-i v_{3}
\end{array}\right)
$$


Dually, the tensor product $S^{A A^{\prime}}=S^{A} \otimes S^{A^{\prime}}$ is isomorphic to the space of vectors $V^{a}$. In the same way, the gradient $\nabla_{a}, a=0,1,2,3$, corresponds to

$$
\nabla_{A A^{\prime}}=\left(\begin{array}{cc}
\nabla_{00^{\prime}} & \nabla_{01^{\prime}} \\
\nabla_{10^{\prime}} & \nabla_{11^{\prime}}
\end{array}\right) \simeq \frac{1}{\sqrt{2}}\left(\begin{array}{cc}
\partial_{0}+i \partial_{3} & \partial_{1}+i \partial_{2} \\
-\partial_{1}+i \partial_{2} & \partial_{0}-i \partial_{3}
\end{array}\right)
$$

Useful relations are

$$
x_{A A^{\prime}} x^{B A^{\prime}}=\varepsilon_{A}{ }^{B}|x|^{2} \text { and } \nabla_{A A^{\prime}} \nabla^{B A^{\prime}}=\varepsilon_{A}{ }^{B} \Delta
$$

where $|x|^{2}=\left|v_{0}\right|^{2}+\left|v_{1}\right|^{2}+\left|v_{2}\right|^{2}+\left|v_{3}\right|^{2}$ and $\Delta$ is the corresponding Laplacian.

This identification extends to higher order tensor products. In particular, in dimension 4 , the second outer power $\Lambda^{2}\left(E_{c}\right)$ splits into the direct sum

$$
\Lambda^{2}\left(E_{c}\right) \simeq \Lambda_{+}^{2}\left(E_{c}\right) \oplus \Lambda_{-}^{2}\left(E_{c}\right)
$$

of selfdual (SD) and anti-selfdual (ASD) 2-forms. For $s_{A B}=s_{(A B)} \in \odot^{2}\left(S_{A}\right)$, the product $s_{A B} \varepsilon_{A^{\prime} B^{\prime}}$ belongs to $\Lambda_{+}^{2}\left(E_{c}\right)$, and similarly for the primed version.

\section{SPIN-1 FIELDS AND FIRST ORDER INVARIANT OPERATORS}

In this section we will study systems of first order differential operators which are invariant with respect to the group $\operatorname{Spin}(4, \mathbb{C})$ acting on functions defined on (open subsets of) $\mathbb{R}^{4}$, and with values in irreducible complex $\operatorname{Spin}(4, \mathbb{C})$-modules. Up to an isomorphism there are only three irreducible spin 1 modules for $\operatorname{Spin}(4, \mathbb{C})$, viz. $S_{(A B)}, S_{A A^{\prime}}$ and $S_{\left(A^{\prime} B^{\prime}\right)}$.

3.1. Invariant operators. We introduce the following operators defined on spaces $\Gamma$ of smooth maps on $E \simeq \mathbb{R}^{4}$ with values in the corresponding spin 1 modules.

- The differential operator $D^{+}: \Gamma\left(S_{(A B)}\right) \rightarrow \Gamma\left(S_{A A^{\prime}}\right)$ :

$$
D^{+}: \varphi_{A B} \mapsto\left(D^{+} \varphi\right)_{A A^{\prime}}=\nabla_{C A^{\prime}} \varphi_{A B} \varepsilon^{B C}=\nabla_{A^{\prime}}^{B} \varphi_{B A}
$$

is the massless field operator of spin 1, acting on fields with values in $S_{(A B)}$.

- The differential operator $D^{-}: \Gamma\left(S_{\left(A^{\prime} B^{\prime}\right)}\right) \rightarrow \Gamma\left(S_{A A^{\prime}}\right)$ :

$$
D^{-}: \varphi_{A^{\prime} B^{\prime}} \mapsto\left(D^{-} \varphi\right)_{A A^{\prime}}=\nabla_{A C^{\prime}} \varphi_{A^{\prime} B^{\prime}} \varepsilon^{B^{\prime} C^{\prime}}=\nabla_{A}^{B^{\prime}} \varphi_{B^{\prime} A^{\prime}}
$$

is the massless field operator of spin 1 , acting on fields with values in $S_{\left(A^{\prime} B^{\prime}\right)}$.

- The differential operator $E^{+}: \Gamma\left(S_{A A^{\prime}}\right) \mapsto \Gamma\left(S_{(A B)}\right)$ :

$$
\left.E^{+}: \varphi_{A A^{\prime}} \mapsto\left(E^{+} \varphi\right)_{A B}=\nabla_{B^{\prime}(B} \varphi_{A) A^{\prime}} \varepsilon^{A^{\prime} B^{\prime}}=\nabla_{(B}^{A^{\prime}} \varphi_{A}\right) A^{\prime}
$$

is the first of three massless field operators of spin 1, acting on fields with values in $S_{A A^{\prime}}$.

- The differential operator $E^{-}: \Gamma\left(S_{A A^{\prime}}\right) \mapsto \Gamma\left(S_{\left(A^{\prime} B^{\prime}\right)}\right)$ :

$$
E^{-}: \varphi_{A A^{\prime}} \mapsto\left(E^{-} \varphi\right)_{A^{\prime} B^{\prime}}=\nabla_{B\left(B^{\prime}\right.} \varphi_{\left.A^{\prime}\right) A} \varepsilon^{A B}=\nabla_{\left(B^{\prime}\right.}^{A} \varphi_{\left.A^{\prime}\right) A}
$$

is the second massless field operator of spin 1, acting on fields with values in $S_{A A^{\prime}}$.

- The differential operator $F$ from the space of spinor fields with values in $S_{A A^{\prime}}$ to the space of $\mathbb{C}$-valued functions:

$$
F: \varphi_{A A^{\prime}} \mapsto \nabla^{A A^{\prime}} \varphi_{A A^{\prime}}
$$

is the third massless field operator of spin 1 corresponding to the divergence on vector fields.

- The differential operator $G$ from the space of $\mathbb{C}$-valued functions to the space of spinor fields with values in $S_{A A^{\prime}}$ :

$$
G: f \mapsto \nabla_{A A^{\prime}} f
$$

is the operator corresponding to the de Rham differential on functions. 
For $X=D^{ \pm}, E^{ \pm}, F, G$, we denote by $X_{j}$ the operator $X$ restricted to the space of homogeneous polynomials of degree $j \in\{0,1,2, \ldots\}$. With this notation, e.g., the kernel of the massless field operator $D^{+}$is equal to $\bigoplus_{j=0}^{\infty} \operatorname{Ker} D_{j}^{+}$.

3.2. Identification of the spinor fields and forms. As noticed above, the space $V_{a}$ of 1 -forms on $\mathbb{R}^{4}$ is isomorphic to $S_{A A^{\prime}}$. Hence

$$
\Lambda^{2}\left(V_{a}\right) \simeq \Lambda^{2}\left(S_{A} \otimes S_{A^{\prime}}\right) \simeq\left[\odot^{2}\left(S_{A}\right) \otimes \Lambda^{2}\left(S_{A^{\prime}}\right)\right] \oplus\left[\odot^{2}\left(S_{A^{\prime}}\right) \otimes \Lambda^{2}\left(S_{A}\right)\right] .
$$

But $\Lambda^{2}\left(S_{A}\right) \simeq \Lambda^{2}\left(S_{A^{\prime}}\right) \simeq \mathbb{C}$, so we obtain the following statement.

Lemma 3.1. Let $\mathcal{E}^{j}, j=0,1,2$, denote the space of smooth differential $j$-forms on $\mathbb{R}^{4}$ and let $\mathcal{E}_{ \pm}^{2}$ denote the spaces of selfdual, respectively anti-selfdual 2-forms. Then

$$
\begin{gathered}
\mathcal{C}^{\infty}\left(\mathbb{R}^{4}, S_{A B}\right) \simeq \mathcal{E}_{+}^{2}, \quad \mathcal{C}^{\infty}\left(\mathbb{R}^{4}, S_{A^{\prime} B^{\prime}}\right) \simeq \mathcal{E}_{-}^{2}, \\
\mathcal{C}^{\infty}\left(\mathbb{R}^{4}, S_{A A^{\prime}}\right) \simeq \mathcal{E}^{1}, \quad \mathcal{C}^{\infty}\left(\mathbb{R}^{4}, \mathbb{C}\right) \simeq \mathcal{E}^{0} .
\end{gathered}
$$

Consequently we obtain an interpretation of the invariant differential operators between spinor fields in terms of de Rham differentials (resp. co-differentials). The operators $D^{ \pm}$ are the restriction of the co-differential $d^{*}$ to the space of selfdual (resp. anti-selfdual) 2 -forms. The operators $E^{ \pm}$are the projection of the differential $d$ acting on 1 -forms to $\mathcal{E}_{ \pm}^{2}$. Finally, the operator $G$ is the de Rham differential on functions and the operator $F$ is the co-differential $d^{*}$ acting on 1 -forms.

3.3. The Fischer inner product. In order to study the Fischer decomposition we need a version of the traditional Fischer inner product in the present context. Let $\mathcal{P} o \ell$ denote the space of complex-valued polynomials in $\mathbb{R}^{4}$ and let $x^{\alpha}$ be the basis of $\mathcal{P}$ ol given by $x^{\alpha}=x_{1}^{\alpha_{1}} \ldots x_{4}^{\alpha_{4}}$. For the multi-index $\alpha=\left(\alpha_{1}, \ldots, \alpha_{4}\right)$, let $\alpha !=\alpha_{1} ! \ldots \alpha_{4} !$. The standard definition of the Fischer inner product on the space $\mathcal{P} o l$ then is

$$
(p(x), q(x)):=[p(\partial)(q(x))]_{x=0}, \quad p, q \in \mathcal{P} \text { ol }
$$

where $p(\partial)$ denotes the constant coefficient differential operator obtained by substituting derivatives $\partial_{i}$ for the variables $x_{i}$. An alternative definition is obtained by declaring the basis $x^{\alpha}$ to be orthogonal, with $\left(x^{\alpha}, x^{\alpha}\right)=\alpha$ !. As an immediate corollary we obtain

$$
\left(x_{a} p(x), q(x)\right)=\left(p(x), \partial_{a} q(x)\right), \quad p, q \in \mathcal{P} \text { ol }
$$

It is well known that the Fischer inner product is invariant with respect to the action of the orthogonal group. This immediately follows from another equivalent definition given by the $L_{2}$ scalar product of the polynomials $p$ and $q$ restricted to the unit sphere. The latter scalar product differs from the Fischer scalar product on the space of homogeneous polynomials of a given degree just by a constant multiple (for details, see [5]).

To extend the Fischer scalar product to spinor-valued fields, we use the invariant scalar product on the spinor spaces and on their tensor products.

Definition 3.2. The (complex valued) scalar product is defined for decomposable elements in the spinor field and for $p, q \in \mathcal{P}$ ol by

$$
\begin{aligned}
\left(\phi_{A A^{\prime}} p(x), \psi_{A A^{\prime}} q(x)\right) & =\phi^{A A^{\prime}} \psi_{A A^{\prime}}(p(x), q(x)) ; \phi_{A A^{\prime}}, \psi_{A A^{\prime}} \in S_{A A^{\prime}} \\
\left(\phi_{A B} p(x), \psi_{A B} q(x)\right) & =\phi^{A B} \psi_{A B}(p(x), q(x)) ; \phi_{A B}, \psi_{A B} \in S_{A B} \\
\left(\phi_{A^{\prime} B^{\prime}} p(x), \psi_{A^{\prime} B^{\prime}} q(x)\right) & =\phi^{A^{\prime} B^{\prime}} \psi_{A^{\prime} B^{\prime}}(p(x), q(x)) ; \phi_{A^{\prime} B^{\prime}}, \psi_{A^{\prime} B^{\prime}} \in S_{A^{\prime} B^{\prime}}
\end{aligned}
$$

and is extended by linearity to the whole tensor product $\mathcal{P}$ ol $\left(S_{A A^{\prime}}\right):=\mathcal{P}$ ol $\otimes S_{A A^{\prime}}$, $\mathcal{P} O \ell\left(S_{A B}\right):=\mathcal{P} O \ell \otimes S_{A B}$ and $\mathcal{P} O \ell\left(S_{A^{\prime} B^{\prime}}\right):=\mathcal{P} O \ell \otimes S_{A^{\prime} B^{\prime}}$ respectively. 
3.4. Invariant algebraic operators. In subsection 3.1 we have introduced several invariant differential operators acting on spin 1 spinor fields. Their duals with respect to the Fischer inner product, denoted by $\left(E^{ \pm}\right)^{*},\left(D^{ \pm}\right)^{*}, F^{*}$ and $G^{*}$ respectively, are given by linear functions acting on spinor fields by multiplication. This duality is expressed by the following relations.

Lemma 3.3. The following identities hold:

$$
\begin{aligned}
\left(x_{A^{\prime}}^{B} \varphi_{B A}(x), \psi_{A A^{\prime}}(x)\right) & =\left(\varphi_{A B}(x),-\left[E^{+}(\psi)\right]_{A B}(x)\right) \\
\left(x_{A}^{B^{\prime}} \varphi_{B^{\prime} A^{\prime}}(x), \psi_{A A^{\prime}}(x)\right) & =\left(\varphi_{A^{\prime} B^{\prime}}(x),-\left[E^{-}(\psi)\right]_{A^{\prime} B^{\prime}}(x)\right) \\
\left(x_{(B}^{A^{\prime}} \varphi_{A) A^{\prime}}(x), \psi_{A B}(x)\right) & =\left(\varphi_{A A^{\prime}}(x),-\left[D^{+}(\psi)\right]_{A A^{\prime}}(x)\right) \\
\left(x_{\left(B^{\prime}\right.}^{A} \varphi_{\left.A^{\prime}\right) A}(x), \psi_{A^{\prime} B^{\prime}}(x)\right) & =\left(\varphi_{A A^{\prime}}(x),-\left[D^{-}(\psi)\right]_{A A^{\prime}}(x)\right) \\
\left(x_{A A^{\prime}} \varphi(x), \psi_{A A^{\prime}}(x)\right) & =(\varphi(x),[F(\psi)](x)) \\
\left(x^{A A^{\prime}} \varphi_{A A^{\prime}}(x), \psi(x)\right) & =\left(\varphi_{A A^{\prime}}(x),[G(\psi)]_{A A^{\prime}}(x)\right)
\end{aligned}
$$

Proof. Let us prove the first identity, the proof of the other ones being similar. It is sufficient to consider decomposable elements:

$$
\begin{aligned}
\left(x_{A^{\prime}}^{B} \varphi_{B A} p(x), \psi_{A A^{\prime}} q(x)\right) & =\left(\sigma_{A^{\prime} a}^{B} x^{a} \varphi_{B A} p(x), \psi^{A A^{\prime}} q(x)\right) \\
& =\left(\sigma_{A^{\prime} a}^{B} \varphi_{B A}\right) \psi^{A^{\prime} A}\left(x^{a} p(x), q(x)\right) \\
& =\varphi_{B A}\left(\sigma_{A^{\prime} a}^{B} \psi^{A A^{\prime}}\right)\left(p(x), \partial^{a} q(x)\right) \\
& =\left(\varphi_{B A} p(x), \nabla_{A^{\prime} B}\left(\psi_{A}^{A^{\prime}} q(x)\right)\right) \\
= & \left(\varphi_{A B}(x),-\left[E^{+}(\psi)\right]_{A B}(x)\right)
\end{aligned}
$$

\section{Polynomial SPIN 1 FIEldS}

We decompose the space of complex-valued polynomials as $\mathcal{P} O \ell=\oplus_{k=0}^{+\infty} P_{k}$, where the space $P_{k}$ of complex-valued $k$-homogeneous polynomials in $\mathbb{R}^{4}$ can itself be decomposed into irreducible $\mathfrak{s o}_{4} \mathbb{C}$-modules as

$$
P_{k}=\bigoplus_{j=0}^{\left[\frac{k}{2}\right]}|x|^{2 j} \mathcal{H}_{k-2 j},
$$

where $\mathcal{H}_{j}$ is the space of complex-valued spherical harmonics of degree $j$, in our notation corresponding to $(j, j)$. The tensor product $P_{k} \otimes S$ decomposes into irreducible subrepresentations of $\mathfrak{s o}_{4} \mathbb{C}$ which can be grouped according to the subspaces in the decomposition (6).

Lemma 4.1. For all $k \geq 0$ the $\mathfrak{s o}_{4} \mathbb{C}$-module $P_{k} \otimes S_{(A B)}$ decomposes into irreducible components as

$$
P_{k} \otimes S_{(A B)}=\bigoplus_{j=0}^{\left[\frac{k}{2}\right]}|x|^{2 j} \mathcal{H}_{k-2 j} \otimes S_{(A B)}
$$

where

$$
\begin{aligned}
& \mathcal{H}_{0} \otimes S_{(A B)}=(2,0) \\
& \mathcal{H}_{1} \otimes S_{(A B)}=(3,1) \oplus(1,1), \\
& \mathcal{H}_{j} \otimes S_{(A B)}=(j+2, j) \oplus(j, j) \oplus(j-2, j) \quad \text { for } j \geq 2 .
\end{aligned}
$$

Proof. The statement immediately follows from the Clebsch-Gordan formula

$$
(c) \otimes(d)=(c+d) \oplus \cdots \oplus(|c-d|)
$$

applied to every component separately. 
Let us illustrate Lemma 4.1 for the case $k=2$. We have

$$
\mathcal{H}_{2} \otimes S_{(A B)}=(2,2) \otimes(2,0)=((2) \otimes(2),(2) \otimes(0))=(4,2) \oplus(2,2) \oplus(0,2)
$$

leading to

$$
P_{2} \otimes S_{(A B)}=\left(\mathcal{H}_{0} \otimes S_{(A B)}\right) \oplus\left(\mathcal{H}_{2} \otimes S_{(A B)}\right)=(2,0) \oplus(0,2) \oplus(2,2) \oplus(4,2)
$$

The statement of Lemma 4.1 is visualised in Scheme 1. The column with label $k$ contains all irreducible subrepresentations in the decomposition of $P_{k} \otimes S_{(A B)}$. The horizontal arrows correspond to the multiplication by $|x|^{2}$ which maps every $(i, j)$ into $(i, j)$.

Scheme 1

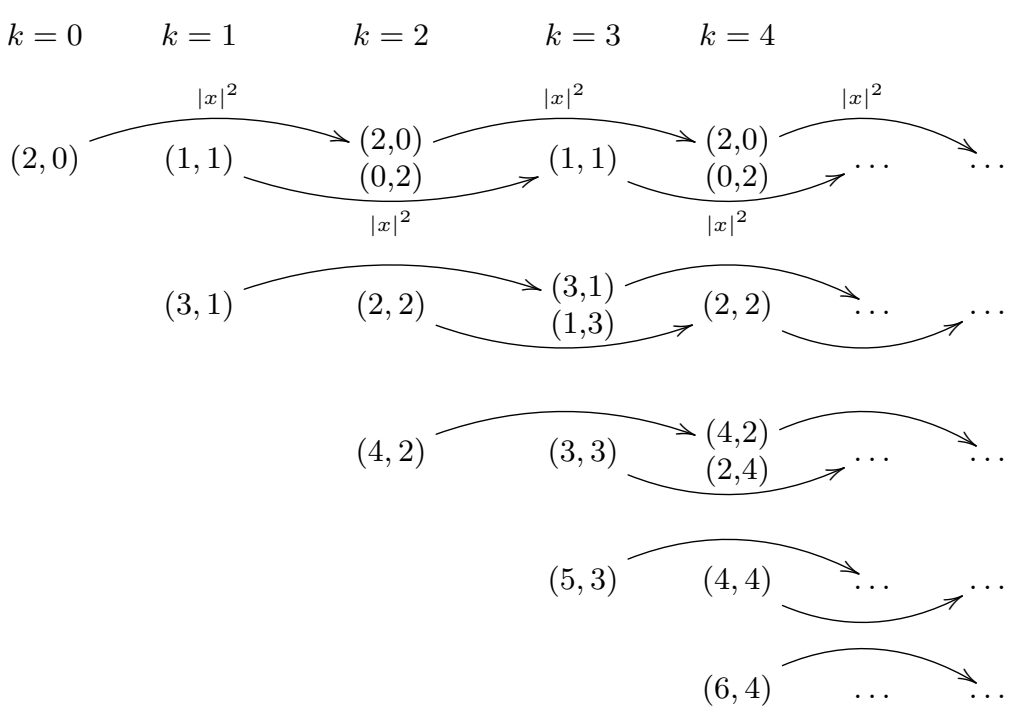

Completely similarly the dual lemma can be proved and visualised, see Scheme 2.

Lemma 4.2. For all $k \geq 0$ the $\mathfrak{s o}_{4} \mathbb{C}$-module $P_{k} \otimes S_{\left(A^{\prime} B^{\prime}\right)}$ decomposes into irreducible components as

$$
P_{k} \otimes S_{\left(A^{\prime} B^{\prime}\right)}=\bigoplus_{j=0}^{\left[\frac{k}{2}\right]}|x|^{2 j} \mathcal{H}_{k-2 j} \otimes S_{\left(A^{\prime} B^{\prime}\right)}
$$

where

$$
\begin{aligned}
& \mathcal{H}_{0} \otimes S_{\left(A^{\prime} B^{\prime}\right)}=(0,2), \\
& \mathcal{H}_{1} \otimes S_{\left(A^{\prime} B^{\prime}\right)}=(1,3) \oplus(1,1), \\
& \mathcal{H}_{j} \otimes S_{\left(A^{\prime} B^{\prime}\right)}=(j, j+2) \oplus(j, j) \oplus(j, j-2) \quad \text { for } j \geq 2 \text {. }
\end{aligned}
$$




\section{Scheme 2}

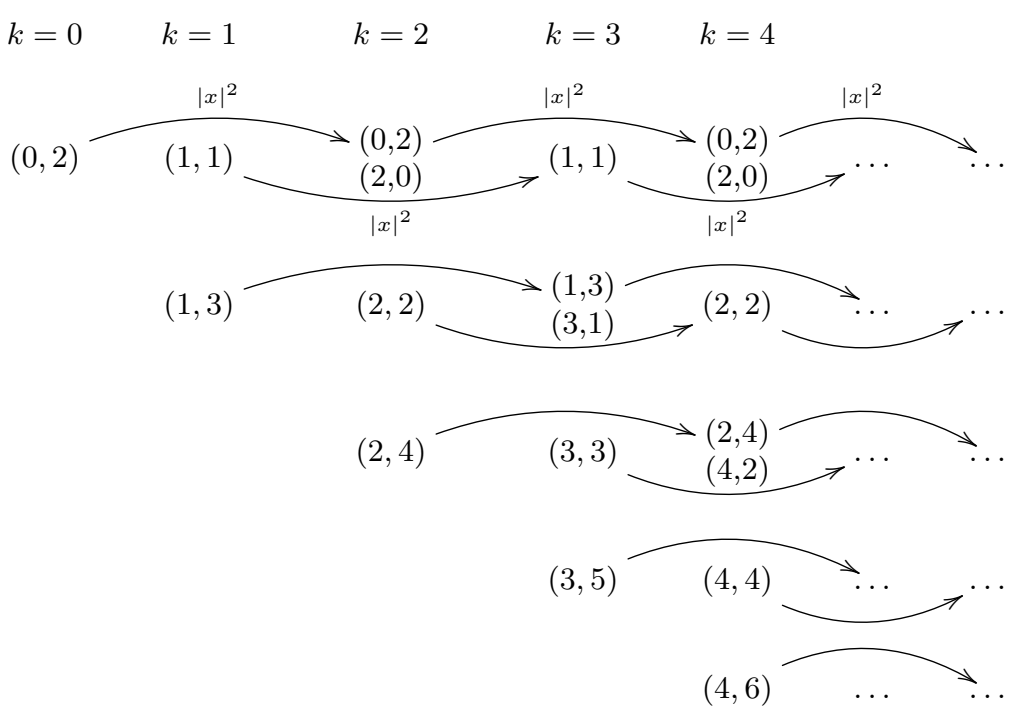

Finally also the following lemma can be proven along the same lines; it is visualised in Scheme 3.

Lemma 4.3. For all $k \geq 0$ the $\mathfrak{s o}_{4} \mathbb{C}$-module $P_{k} \otimes S_{A B^{\prime}}$ decomposes into irreducible components as

$$
P_{k} \otimes S_{A B^{\prime}}=\bigoplus_{j=0}^{\left[\frac{k}{2}\right]}|x|^{2 j} \mathcal{H}_{k-2 j} \otimes S_{A B^{\prime}} \quad \text { for every } k \geq 0
$$

where

$$
\begin{aligned}
\mathcal{H}_{0} \otimes S_{A B^{\prime}}= & (1,1), \\
\mathcal{H}_{j} \otimes S_{A B^{\prime}}= & (j+1, j+1) \oplus(j+1, j-1) \oplus(j-1, j+1) \oplus(j-1, j-1) \\
& \quad \text { for } j \geq 1 .
\end{aligned}
$$




\section{Scheme 3}

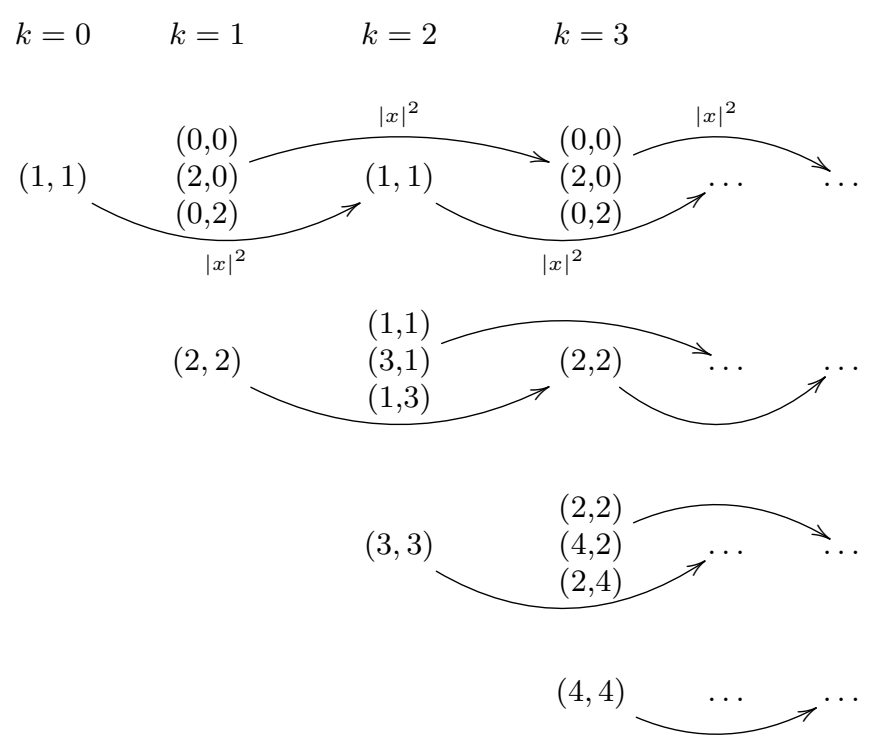

\section{THE KERNEL OF THE MASSLESS FIELD OPERATORS}

In order to obtain the Fischer decomposition aimed at it is essential to unravel the structure of the kernel of the massless field operators in the three cases considered. First we treat the case of selfdual fields.

Lemma 5.1. For all $k=0,1,2, \ldots$ the kernel of the operator $D_{k}^{+}$acting on the space $P_{k} \otimes S_{(A B)}$ is an irreducible Spin(4)-module isomorphic to $(k+2, k)$.

Proof. We may assume that $k \geq 1$. We proceed stepwise.

(i) It holds that $(k+2, k) \subset \operatorname{Ker} D_{k}^{+}$. Indeed, the operator $D_{k}^{+}$intertwines with the action of Spin(4) and has values in $P_{k-1} \otimes S_{A^{\prime} B}$. It is seen that there is no component isomorphic to $(k+2, k)$ in its decomposition, hence the (unique) piece in $P_{k} \otimes S_{(A B)}$ isomorphic to $(k+2, k)$ belongs to the kernel of $D_{k}^{+}$.

(ii) Every space of the form $\operatorname{Ker} D_{k}^{+}$is a subspace of $\mathcal{H}_{k} \otimes S_{(A B)}=(k+2, k) \oplus(k, k) \oplus$ $(k-2, k)$. Indeed, if a field $\varphi_{A B}$ belongs to $\operatorname{Ker} D_{k}^{+}$, then

$$
\nabla_{A A^{\prime}} \nabla^{C A^{\prime}} \varphi_{C B}=\varepsilon_{A}^{C} \Delta \varphi_{C B}=\Delta \varphi_{A B}=0 .
$$

So there are at most two other components in $\operatorname{Ker} D_{k}^{+}$.

(iii) Now we show that $(k, k) \oplus(k-2, k)$ is not a subset of $\operatorname{Ker} D_{k}^{+}$. From the relation

$$
\left(D^{+}\left(\phi_{A B}, \psi^{B A^{\prime}}\right)=\left(\phi_{A B},-x_{(A}^{A^{\prime}} \psi_{B) A^{\prime}}\right)=0,\right.
$$

it follows that the kernel of $D^{+}$is orthogonal to the image of the map $\left(D^{+}\right)^{*}$ given by multiplication with $x^{A A^{\prime}}$. But the components isomorphic to $(k, k)$ and $(k, k-2)$ are images of the corresponding components in $P_{k-1} \otimes S_{B A^{\prime}}$ with respect to $\left(D^{+}\right)^{*}$, hence they cannot be in the kernel of $D_{k}^{+}$. 
In order to make the proof of Lemma 5.1 more transparent, we show below the scheme of this decomposition (Scheme 4). The scheme consists of a cluster of three Fischer-like layers connected by arrows explained below. The symbol $(a, b)$ denotes a space of functions as used above for the corresponing spaces of polynomials with the indicated values. Each column corresponds to a homogeneity degree $k=0,1,2 \ldots$; kernels of $D_{k}^{+}$are shown in full frames, while the dashed frames are the intersections of the kernels $E_{k}^{ \pm}$and $F_{k}$, and double frames are kernels of the dual massless field operator $D_{k}^{-}$. We only draw arrows whose results are in the Fischer decomposition of $D$, i.e. arrows labelled ' $|x|^{2}$ ' going to the right, which denote the multiplication by $|x|^{2}$, and arrows going upwards, from a lower layer to an upper one, which denote the multiplicative action of the dual operator $\left(D^{+}\right)^{*}$.

In a similar way the following lemma is obtained and the corresponding diagram is shown in Scheme 5.

Lemma 5.2. For all $k=0,1,2, \ldots$ one has

$$
\operatorname{Ker} D_{k}^{-}=(k, k+2)
$$

For the third case of spin 1 massless fields, we need the intersection of several kernels. The proof is illustrated in Scheme 6.

Lemma 5.3. For $k=0,1,2, \ldots$ one has

$$
\operatorname{Ker} E_{k}^{+} \cap \operatorname{Ker} E_{k}^{-} \cap \operatorname{Ker} F_{k}=(k+1, k+1)
$$

Proof. Let us consider the case $k \geq 1$. We again proceed stepwise.

(i) The space $(k+1, k+1)$ is in the kernel of all three operators, because there is no suitable space of homogeneity degree one less.

(ii) Again, elements in the kernel of all three operators should be in the kernel of the Laplacian $\Delta$. But $\operatorname{Ker} \Delta \simeq(k+2, k) \oplus(k, k+2) \oplus(k, k) \oplus(k+1, k+1)$.

(iii) Now the first two summands are in the image of the multiplication by $x_{A A^{\prime}}$ from the space $P_{k-1} \otimes S_{A B}$, resp. $P_{k-1} \otimes S_{A^{\prime} B^{\prime}}$, hence they cannot be in the kernel.

So we are left to show that also the component $(k, k)$ cannot be in the kernel. This component appears in the space $P_{k} \otimes S_{A A^{\prime}}$ with multiplicity 2. It appears also (with multiplicity 1) in $P_{k-2} \otimes S_{A A^{\prime}}$. Consider a field $\varphi_{A A^{\prime}} \in P_{k-2} \otimes S_{A A^{\prime}}$. We can act on this component in degree $k-2$ in different ways: one is $\left(E^{+}\right)^{*} \circ\left(D^{+}\right)^{*} \varphi$, (or the similar one with minus superscripts), the other one is $F^{*} \circ G^{*} \varphi$. Both maps are intertwining and are defined on an irreducible representation, so either they coincide, or they generate the whole double isotypic component. If the images coincide, we can act on both sides with multiplication by $x_{A A^{\prime}}$ and we obtain

$$
\begin{aligned}
x_{A A^{\prime}}\left(x^{B B^{\prime}} \varphi_{B B^{\prime}}\right) & =x_{A^{\prime}}^{B} x_{(B}^{C^{\prime}} \varphi_{A) C^{\prime}} \\
x_{C}^{A^{\prime}} x_{A A^{\prime}}\left(x^{B B^{\prime}} \varphi_{B B^{\prime}}\right) & =x_{C}^{A^{\prime}} x_{A^{\prime}}^{B} x_{(B}^{C^{\prime}} \varphi_{A) C^{\prime}} \\
|x|^{2} \varepsilon_{C A}\left(x^{B B^{\prime}} \varphi_{B B^{\prime}}\right) & =|x|^{2} \varepsilon_{C}^{B} x_{(B}^{C^{\prime}} \varphi_{A) C^{\prime}}=|x|^{2} x_{(C}^{C^{\prime}} \varphi_{A) C^{\prime}}
\end{aligned}
$$

The spinor on the left hand-side is antisymmetric in $A, C$, while the right hand-side is symmetric in $A, C$, hence both sides vanish. However, there is a choice of $x$ and $\varphi$ for which $x^{B B^{\prime}} \varphi_{B B^{\prime}}$ is nontrivial, which is a contradiction. 
FISCHER DECOMPOSITION FOR MASSLESS FIELDS OF SPIN 1 IN DIMENSION 4

Scheme 4

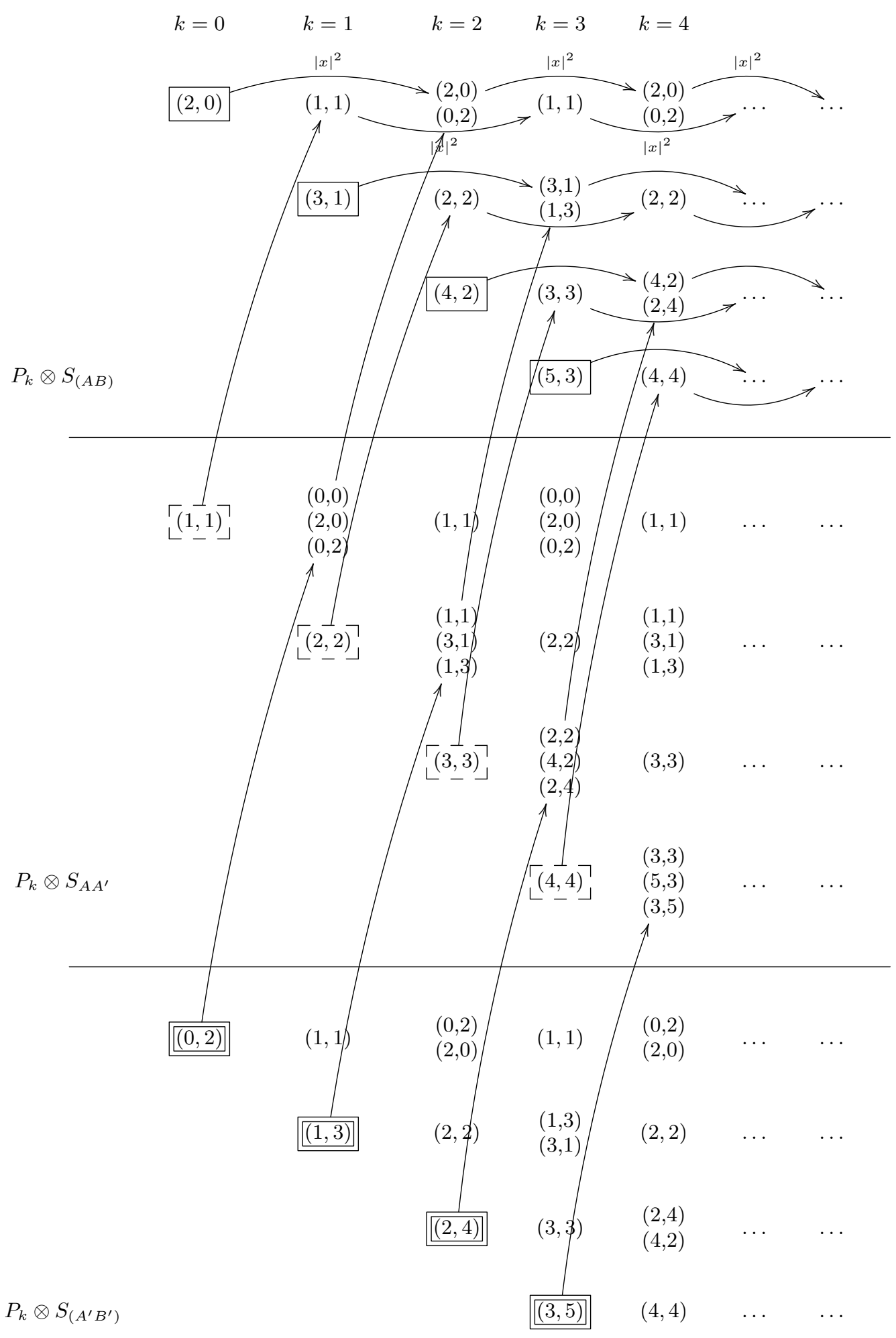




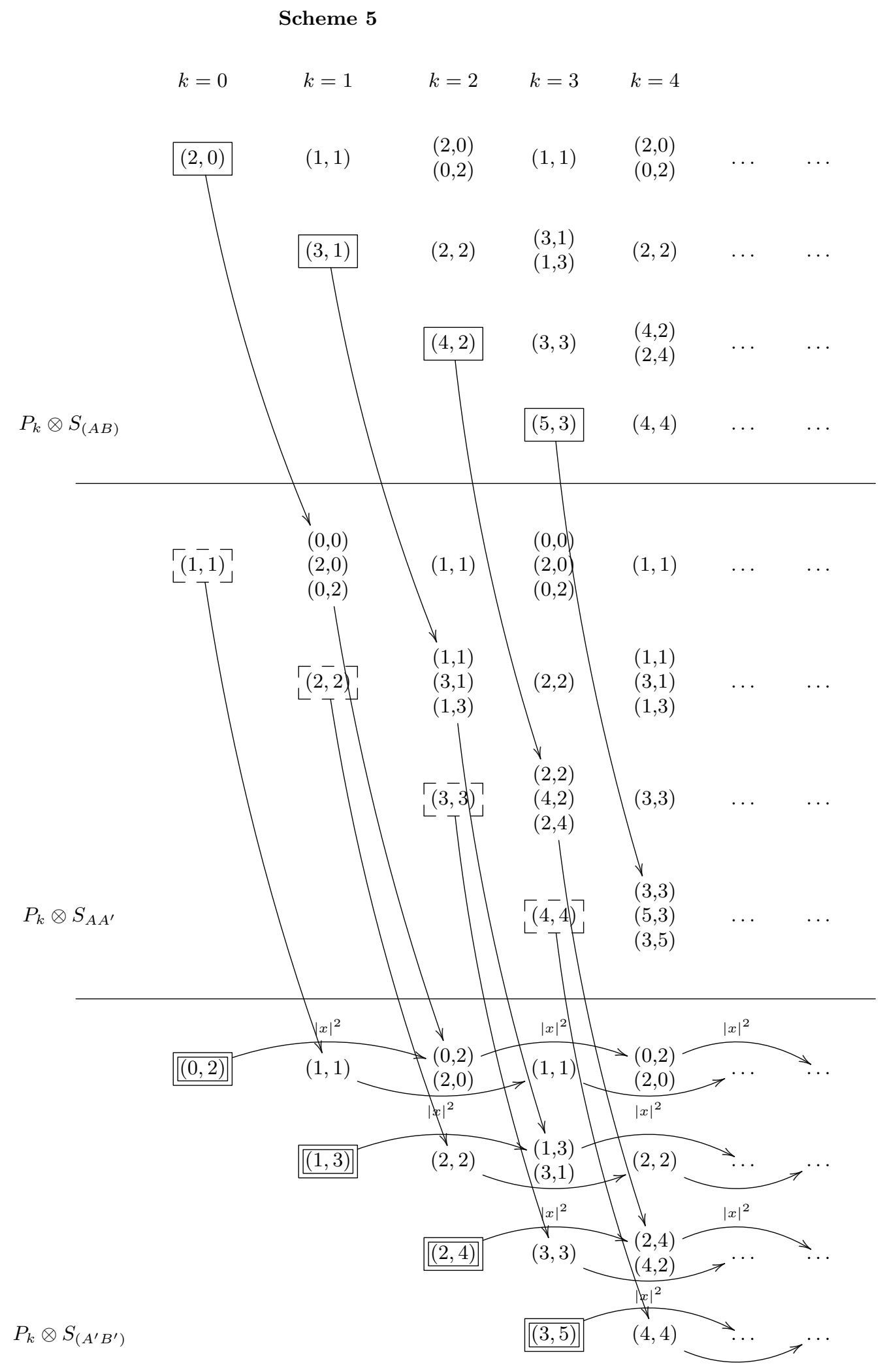


FISCHER DECOMPOSITION FOR MASSLESS FIELDS OF SPIN 1 IN DIMENSION $4 \quad 13$

Scheme 6

$$
\begin{array}{lll}
k=0 & k=1 & k=2 \quad k=3 \\
(0,0) & (0,0)
\end{array}
$$

$(1,1)$

0 - forms

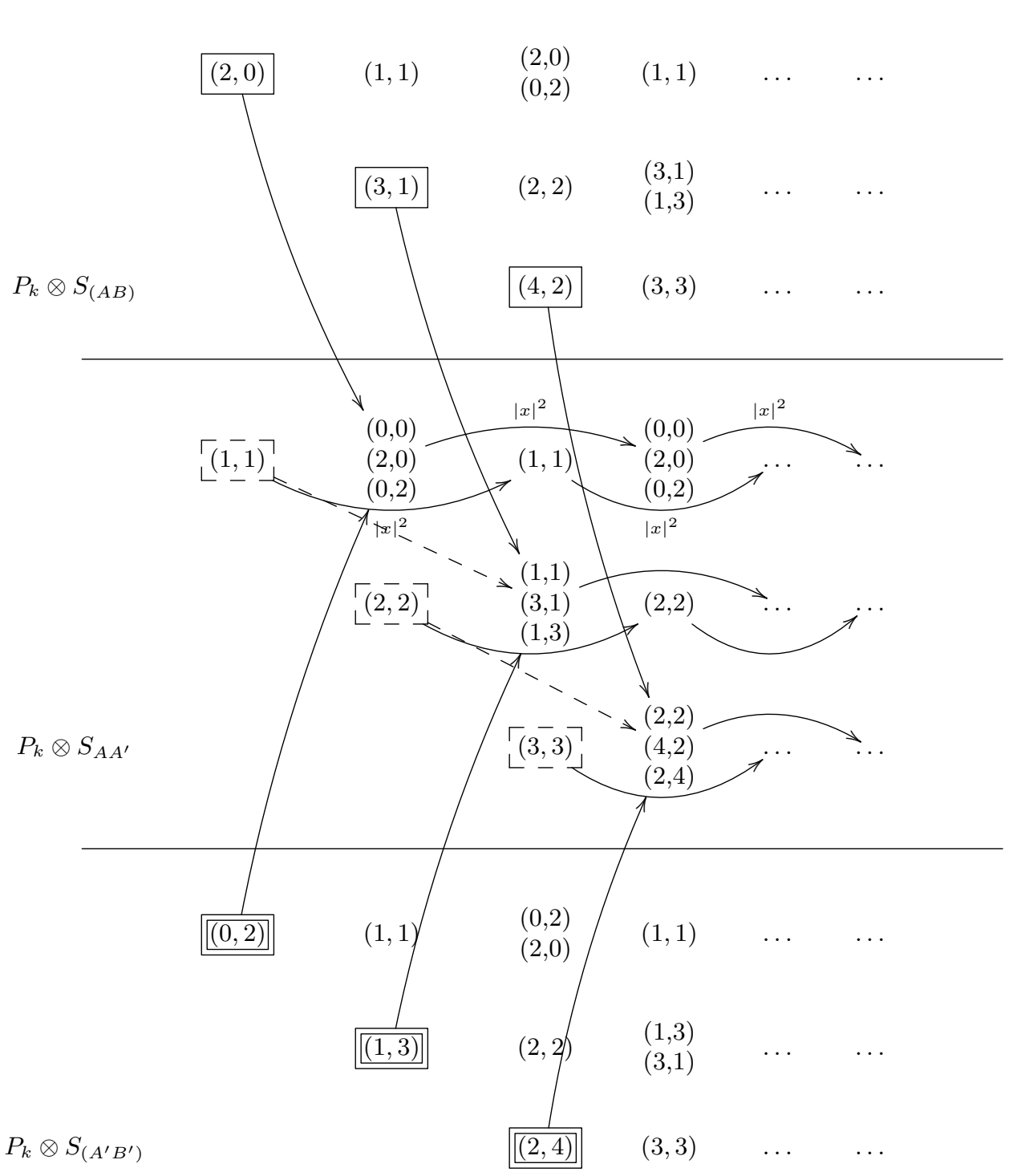

$(2,2)$
$(1,1)$

․ $\quad \ldots$

$\cdots \quad \cdots$ 


\section{The Fischer DeCOMPosition}

The results obtained in the preceding section directly lead to the corresponding theorems on the Fischer decomposition. In each case we know the full decomposition of the spaces of spinor-valued polynomials at the left hand-side, and so it is sufficient to observe that every summand at the right hand-side is a nontrivial irreducible module with the correct representation behaviour for the corresponding homogeneities.

Theorem 6.1. [Fischer decomposition for selfdual massless fields of spin 1] The space of homogeneous polynomials of degree $k$ with values in $S_{(A B)}$ decomposes as:

$$
\begin{aligned}
P_{k} \otimes S_{(A B)}= & \bigoplus_{j=0}^{\left[\frac{k}{2}\right]}|x|^{2 j} \operatorname{Ker} D_{k-2 j}^{+} \\
& \oplus \bigoplus_{j=0}^{\left[\frac{k}{2}\right]}|x|^{2 j}\left(D^{+}\right)^{*}\left(\operatorname{Ker} E_{k-2 j-1}^{+} \cap \operatorname{Ker} E_{k-2 j-1}^{-} \cap \operatorname{Ker} F_{k-2 j-1}\right) \\
& \oplus \bigoplus_{j=0}^{\left[\frac{k}{2}\right]-1}|x|^{2 j}\left(D^{+}\right)^{*} \circ\left(E^{-}\right)^{*}\left(\operatorname{Ker} D_{k-2 j-2}^{-}\right) .
\end{aligned}
$$

The decomposition in Theorem 6.1 of the space $P_{k}\left(S_{(A B)}\right)$ of $k$-homogeneous polynomials is seen in the upper layer of Scheme 4. At its diagonal, in the $k$-th column, there are kernels of $D_{k}^{+}$; at the first parallel to the diagonal there are images of the intersection of kernels of $E_{k}^{ \pm}$and $F_{k}$ by $\left(D^{+}\right)^{*}$, and at the second parallel there are spaces $|x|^{2} \operatorname{Ker} D_{k-2}$ and images $\left(D^{+}\right)^{*} \circ\left(E^{-}\right)^{*}\left(\operatorname{Ker} D_{k-2}^{-}\right)$. At every next position in a row, in the $k$-th column, there is the image of the space in the $(k-2)$-th column under multiplication by $|x|^{2}$.

Theorem 6.2. [Fischer decomposition for anti-selfdual massless fields of spin 1 ] The space of homogeneous polynomials of degree $k$ with values in $S^{\left(A^{\prime} B^{\prime}\right)}$ decomposes as:

$$
\begin{aligned}
P_{k} \otimes S_{\left(A^{\prime} B^{\prime}\right)}= & \bigoplus_{j=0}^{\left[\frac{k}{2}\right]}|x|^{2 j} \operatorname{Ker} D_{k-2 j}^{-} \\
& \oplus \bigoplus_{j=0}^{\left[\frac{k}{2}\right]}|x|^{2 j}\left(D^{-}\right)^{*}\left(\operatorname{Ker} E_{k-2 j-1}^{+} \cap \operatorname{Ker} E_{k-2 j-1}^{-} \cap \operatorname{Ker} F_{k-2 j-1}\right) \\
& \oplus \bigoplus_{j=0}^{\left[\frac{k}{2}\right]-1}|x|^{2 j}\left(D^{-}\right)^{*} \circ\left(E^{+}\right)^{*}\left(\operatorname{Ker} D_{k-2 j-2}^{+}\right) .
\end{aligned}
$$

Theorem 6.3. [Fischer decomposition for the selfdual part of the Hodge-de Rham system] The space of homogeneous polynomials of degree $k$ with values in $S_{A}^{A^{\prime}}$ decomposes as:

$$
\begin{aligned}
P_{k} \otimes S_{A A^{\prime}}= & \bigoplus_{j=0}^{\left[\frac{k}{2}\right]}|x|^{2 j}\left(E^{+}\right)^{*}\left(\operatorname{Ker} D_{k-2 j-1}^{+}\right) \oplus \bigoplus_{j=0}^{\left[\frac{k}{2}\right]}|x|^{2 j}\left(E^{-}\right)^{*}\left(\operatorname{Ker} D_{k-2 j-1}^{-}\right) \\
& \oplus \bigoplus_{j=0}^{\left[\frac{k}{2}\right]}|x|^{2 j}\left(E^{-}\right)^{*} \circ\left(D^{+}\right)^{*}\left(\operatorname{Ker} E_{k-2 j-2}^{+} \cap \operatorname{Ker} E_{k-2 j-2}^{-} \cap \operatorname{Ker} F_{k-2 j-2}\right) \\
& \oplus \bigoplus_{j=0}^{\left[\frac{k}{2}\right]}|x|^{2 j}\left(E^{+}\right)^{*} \circ\left(D^{-}\right)^{*}\left(\operatorname{Ker} E_{k-2 j-2}^{+} \cap \operatorname{Ker} E_{k-2 j-2}^{-} \cap \operatorname{Ker} F_{k-2 j-2}\right) .
\end{aligned}
$$




\section{Conclusion}

We have proven the Fischer decomposition in terms of $\mathfrak{s o}_{4} \mathbb{C}$-irreducible representations for spaces of polynomial solutions to the spin 1 massless field equation in dimension 4 , both in the selfdual and the anti-selfdual cases and for the selfdual part of the Hodge-de Rham system. This is a fundamental contribution to the study of the solutions to the spin 1 massless field equations in 4-dimensional Euclidean space, which are equivalent to the Maxwell equations in the Euclidean setting. The proofs are given in a completely independent way and using the language of spinor fields. The higher spin cases, still in dimension 4 , are subject for further research.

\section{ACKnowledgement}

Lukáš Krump and Vladimír Souček gratefully acknowledge support by the Czech grant GA CR 17-01171S.

\section{REFERENCES}

[1] S. Böck, Über funktionentheoretische Methoden in der räumlichen Elastizitätstheorie, $\mathrm{PhD}$ thesis, Bauhaus-University Weimar, 2009

(url: http://e-pub.uniweimar.de/frontdoor.php?source opus=1503, date: 07.04.2010).

[2] S. Böck, K. Gürlebeck, R. Lávička, V. Souček, Gel'fand-Tsetlin bases for spherical monogenic in dimension 3, Rev. Mat. Iberoamericana 28 (4) (2012), 1165-1192.

[3] F. Brackx, H. De Schepper, L. Krump, V. Souček, Selfdual 2-forms in Dimension 4 and thezir Fischer Decomposition, AIP Conf. Proc. 1479 (296) (2012), 296-299

[4] I. Cacao, H. R. Malonek, On a complete set of hypercomplex Appell polynomials, in: T.E. Simos, G. Psihoyios, Ch. Tsitouras (Eds.), Numerical Analysis and Applied Mathematics, AIP Conference Proceedings 1048, American Institute of Physics, Melville, NY, 2008, 647650.

[5] H. De Bie, F. Sommen, M. Wutzig, Reproducing kernels for polynomial null-solutions of Dirac operators, arXiv:1503.03969v1.

[6] R. Delanghe, R. Lávička, V. Souček, The Gel'fand-Tsetlin bases for Hodge-de Rham systems in Euclidean spaces, Math. Meth. Appl. Sci., 35 (7) (2012), 745-757.

[7] R. Delanghe, R. Lávička, V. Souček, The Fischer decomposition for the Hodge-de Rham systems in Euclidean spaces, Math. Meth. Appl. Sci. 35 (2012), 10-16.

[8] R. Delanghe, R. Lávička, V. Souček, The Howe duality for Hodge systems, In: Proceedings of 18th International Conference on the Application of Computer Science and Mathematics in Architecture and Civil Engineering (ed. K. Gürlebeck and C. Könke), Bauhaus-Universität Weimar, Weimar, 2009.

[9] R. Penrose, M. A. H. MacCallum, Twistor theory. An approach to the quantisation of fields and space-time, Phys. Rep. 6 (1972), 241-316.

[10] R. Penrose, W. Rindler, Spinors and space-times I-II, Cambridge University Press, Cambridge, 1986.

[11] J. Bureš, R.Lávička and V. Souček, Elements of Quaternionic Analysis and Radon Transform, Textos de Matematica, vol. 42, Universidade de Coimbra, Coimbra, 2009 (vi+72 pages, 100 printed copies).

[12] V. Souček, Clifford analysis for higher spins. In F. Brackx et al. (eds.), Clifford Algebras and their Applications in Mathematical Physics, Kluwer, 1993, 223-232.

[13] V. Souček, On massless field equations in higher dimensions. In K. Gürlebeck, C. Könke (eds.), Proc. IKM, 2009, 13 pages.

[14] M. Eastwood, R. Penrose, R.O. Wells, Cohomology and massless fields, Comm. Math. Physics 78 (1981), 305-351. 